\title{
On the mechanism of the so-called uncoupling effect of medium- and short-chain fatty acids

\author{
Peter Schönfeld ${ }^{\text {a }}$, Anna B. Wojtczak ${ }^{b}$, Math J.H. Geelen ${ }^{c}$, Wolfgang Kunz ${ }^{\text {a }}$ \\ and Lech Wojtczak ${ }^{b}$ \\ ${ }^{a}$ Institut für Biochemie der Medizinischen Akademie Magdeburg, Magdeburg (G.D.R.), \\ ${ }^{b}$ Department of Cellular Biochemistry, Nencki Institute of Experimental Biology, Warsaw (Poland) \\ and ' Laboratory of Veterinary Biochemistry, University of Utrecht, Utrecht (The Netherlands)
}

(Received 26 April 1988)

Key words: Medium chain length fatty acid; Uncoupling; Pyruvate carboxylation; Gluconeogenesis; Acyl-CoA hydrolysis; (Mitochondria); (Hepatocytes)

Octanoate applied to rat liver mitochondria respiring with glutamate plus malate or succinate (plus rotenone) under resting-state (State 4) conditions stimulates oxygen uptake and decreases the membrane potential, both effects being sensitive to oligomycin but not to carboxyatractyloside. Octanoate also decreases the rate of pyruvate carboxylation under the same conditions, this effect being correlated with the decrease of intramitochondrial content of ATP and increase of AMP. The decrease of pyruvate carboxylation and the change of mitochondrial adenine nucleotides are both reversed by 2-oxoglutarate. Fatty acids of shorter chain length have similar effects, though at higher concentrations. Addition of octanoate in the presence of fluoride (inhibitor of pyrophosphatase) produces intramitochondrial accumulation of pyrophosphate, even under conditions when oxidation of octanoate is prevented by rotenone. In isolated hepatocytes incubated with lactate plus pyruvate, octanoate also increases oxygen uptake and produces a shift in the profile of adenine nucleotides similar to that observed in isolated mitochondria. It decreases the 'efficiency' of gluconeogenesis, as expressed by the ratio between an increase of glucose production and an increase of oxygen uptake upon addition of gluconeogenic substrates (lactate plus pyruvate), and increases the reduction state of mitochondrial NAD. These effects taken together are not compatible with uncoupling, but point to intramitochondrial hydrolysis of octanoyl-CoA and probably also shorter chain-length acyl-CoAs. This mechanism probably functions as a 'safety valve' preventing a drastic decrease of intramitochondrial free CoA under a large supply of medium- and short-chain fatty acids.

\section{Introduction}

It has long been recognized that fatty acids increase the rate of respiration of perfused liver $[1-5]$ and isolated hepatocytes [6-8]. This effect is

Abbreviations: $\mathrm{DDA}^{+}$, dimethyldibenzylammonium cation; Tricine, $N$-tris(hydroxymethyl)methylglycine.

Correspondence: L. Wojtczak, Nencki Institute of Experimental Biology, Pasteura 3, 02-093 Warsaw, Poland. only partly due to fatty acids being good respiratory substrates for liver, since the increase of respiration produced by fatty acids has also been observed in the presence of excess of other substrates, e.g., lactate or glucose, and can only partly be abolished by phenylaklyl oxirane, inhibitor of carnitine acyltransferase (EG 2.3.1.21) [4]. Although the stimulation of liver respiration by medium- and long-chain fatty acids is well established, attempts to explain this effect are conflicting. 
Since fatty acids are known to uncouple oxidative phosphorylation (for review, see Ref. 9), it seemed plausible to speculate that the increased respiration is due to uncoupling of mitochondrial energy-conserving machinery. This was, however, incompatible with the observation [3] that the stimulation by octanoate and oleate of the respiration of perfused liver was abolished by oligomycin, a known inhibitor of ATP synthesis in mitochondria. It has been therefore proposed [3] that the increased respiration is not evoked by directly dissipating the mitochondrial energy charge but rather by some processes utilizing ATP. More recently, however, the uncoupling hypothesis has been revived by Scholz and co-workers $[4,5]$, who even speculate about a possible physiological role of the 'uncoupling-like' effect of octanoate and oleate.

Working on isolated hepatocytes, Berry et al. [7] proposed that the increased respiration after addition of fatty acids is due to dissipation of the mitochondrial energy charge by reversal of electron transfer in the respiratory chain during ketogenesis. However, the extra oxygen uptake was found to be sensitive to oligomycin $[10,11]$, as in perfused liver [3]. This again argues against the uncoupling or other ways of utilizing the mitochondrial energy state without participation of ATP. Plomp et al. [10] observed that the stimulatory effect of octanoate and oleate on hepatocyte respiration was inhibited $50-70 \%$ by carboxyatractyloside and $50-60 \%$ by ouabain. On that basis, they concluded that fatty acids stimulate extramitochondrial ATP-utilizing process(es) of which the $\mathrm{Na}^{+} / \mathrm{K}^{+}$-ATPase is the main representative. Brand and Nobes [11] found a complete abolishment by carboxyatractyloside of the extra oxygen uptake produced in isolated hepatocytes by octanoate, thus further substantiating the postulate of extramitochondrial increase of ATP turnover.

In view of these conflicting hypotheses and results, we decided to re-investigate the problem of the stimulatory effect of octanoate and other medium- and short-chain fatty acids on oxygen uptake, using both hepatocytes and isolated liver mitochondria.

Preliminary results have been presented elsewhere [12].

\section{Material and Methods}

Mitochondria were isolated from livers of female albino rats (150-200 g body weight) by a standard procedure [13] and suspended in 250 $\mathrm{mM}$ sucrose $/ 5 \mathrm{mM}$ Tris- $\mathrm{HCl}$ ( $\mathrm{pH} 7.4$ ). The protein content of this stock suspension was measured by the biuret method.

Hepatocytes were isolated from male rats $(250-300 \mathrm{~g})$ of the Wistar strain, starved overnight, according to the method of Berry and Friend [14], with the modification described by Geelen and Gibson [15]. Viability of the cells was tested with Trypan blue. Preparations containing more than $90 \%$ of viable cells were used for experiment. Cells (20-30 mg wet weight $/ \mathrm{ml})$ were incubated in the Krebs-Henseleit [16] bicarbonate medium containing $0.85 \mathrm{mM} \mathrm{CaCl}_{2}$ and $1 \%$ defatted and dialysed bovine serum albumin.

The standard incubation medium for mitochondria comprised $110 \mathrm{mM}$ sucrose, $60 \mathrm{mM} \mathrm{KCl}, 60$ $\mathrm{mM}$ Tris- $\mathrm{HCl}$ (pH 7.4), $10 \mathrm{mM}$ potassium phosphate (pH 7.4), $15 \mathrm{mM}$ glucose, $5 \mathrm{mM} \mathrm{MgCl}_{2}$ and $0.5 \mathrm{mM}$ EDTA. Fatty acids were added as sodium salts.

Oxygen uptake by mitochondria was measured polarographically at $25^{\circ} \mathrm{C}$ using a Clark-type electrode and recording either $\mathrm{O}_{2}$ concentration (usual arrangement) or the first derivative of the oxygen concentration trace (a direct measure of the oxygen-uptake rate). The respiration of isolated hepatocytes was determined at $37^{\circ} \mathrm{C}$ in a Gilson respirometer using the vessels and procedure described by Krebs et al. [17].

The membrane potential was determined at $25^{\circ} \mathrm{C}$ using a DDA-sensitive electrode and calculated as described previously [18]. Because of the interference of $\mathrm{K}^{+}$in $\mathrm{DDA}^{+}$determination, the medium for these experiments comprised $90 \mathrm{mM}$ sucrose, $60 \mathrm{mM}$ Tricine- $\mathrm{HCl}, 60 \mathrm{mM}$ Tris- $\mathrm{HCl}, 10$ $\mathrm{mM}$ sodium phosphate, $15 \mathrm{mM}$ glucose, $40 \mathrm{mM}$ nicotinamide, $5 \mathrm{mM} \mathrm{MgCl}$, $0.5 \mathrm{mM}$ EDTA, 3.5 $\mu \mathrm{M}$ tetraphenylborone and $34.5 \mu \mathrm{M}$ DDA bromide added stepwise to calibrate the electrode.

Pyruvate carboxylation was determined by measuring incorporation of $\mathrm{H}^{14} \mathrm{CO}_{3}^{-}$into acidstable metabolites, as described previously [19]. Mitochondria were incubated at $25^{\circ} \mathrm{C}$ in the standard medium containing, in addition, $20 \mathrm{mM}$ 
$\mathrm{KH}^{14} \mathrm{CO}_{3}$ (about $10^{5} \mathrm{dpm} / \mathrm{ml}$ ) and $10 \mathrm{mM}$ pyruvate. $1-\mathrm{ml}$ aliquots were removed after $3,6,9$ and $12 \mathrm{~min}$, acidified with perchloric acid to $6 \%$ final concentration and bubbled for $3 \mathrm{~min}$ with $\mathrm{CO}_{2} \cdot{ }^{14} \mathrm{C}$ was determined by scintillation counting.

ATP [20], ADP and AMP [21], acetoacetate [22] and 3-hydroxybutyrate [23] were determined by enzymatic spectrophotometric procedures and glucose was measured colorimetrically with glucose oxidase (EC 1.1.3.4) and peroxidase (EC 1.11.1.7) [24], in neutralized perchloric extracts from whole suspensions of mitochondria or cells.

The incubations in which intramitochondrial pyrophosphate formation had to be measured were carried out in the medium with phosphate content reduced to $1 \mathrm{mM}$. The aliquots were subjected to silicone oil gradient centrifugation with $0.25 \mathrm{M}$ perchloric acid as the bottom phase. Pyrophosphate was determined in the neutralized extracts as described by Grindey and Nichol [25].

\section{Results}

Short- and medium-chain length fatty acids under resting-state (State 4) conditions increased mitochondrial respiration with NAD-linked substrates (Table I) and succinate plus rotenone (not shown). The effect was dependent on the chain length. Shorter fatty acids appeared less effective, as more of them had to be added in order to obtain the same increase of $\mathrm{O}_{2}$ uptake. Octanoylcarnitine, the readily oxidizable derivative of octanoate, had a negligible effect on mitochondrial respiration. This stimulation of oxygen uptake was not due to uncoupling as it was prevented by oligomycin (Table I and Fig. 1). It was, however, not prevented by carboxyatractyloside (Fig. 1). Another argument against the uncoupling effect of octanoate was provided by measuring its effect on the mitochondrial membrane potential. Although this potential was decreased by octanoate, it was fully restored by oligomycin (Fig. 1).

Octanoate and, to a lesser degree, butyrate and acetate decreased the rate of pyruvate carboxylation in isolated mitochondria (Table II). Analysis of intramitochondrial adenine nucleotides revealed that this decrease was paralleled by a dras-

\section{TABLE I}

EFFECT OF SHORT- AND MEDIUM-CHAIN FATTY ACIDS ON MITOCHONDRIAL RESPIRATION AND ITS REVERSAL BY OLIGOMYCIN

Rat liver mitochondria (1.5-2.0 $\mathrm{mg}$ protein $/ \mathrm{ml})$ were incubated in the standard medium containing glutamate $(5 \mathrm{mM})$ and malate $(5 \mathrm{mM})$ as substrates. Oligomycin was added to the concentration of $5 \mu \mathrm{g} / \mathrm{ml}$. Mean values \pm S.D. for 3-6 different mitochondrial preparations are shown. The significance with respect to the control without added fatty acid, calculated by the Student's $t$-test, was $P<0.05$ where indicated (*).

\begin{tabular}{lcc}
\hline Fatty acid added & $\begin{array}{l}\text { Rate of oxygen uptake } \\
\text { (nmol } \mathrm{O}_{2} / \mathrm{min} \text { per mg protein) }\end{array}$ \\
\cline { 2 - 3 } & $\begin{array}{l}\text { before } \\
\text { oligomycin }\end{array}$ & $\begin{array}{l}\text { after addition } \\
\text { of oligomycin }\end{array}$ \\
\hline None & $8.3 \pm 0.9$ & $6.8 \pm 1.4$ \\
Butyrate $(5.0 \mathrm{mM})$ & $12.0 \pm 0.9^{*}$ & $7.0 \pm 0.8$ \\
Hexanoate $(2.5 \mathrm{mM})$ & $11.8 \pm 0.4^{*}$ & $7.6 \pm 0.8$ \\
Octanoate $(0.5 \mathrm{mM})$ & $13.8 \pm 1.0^{*}$ & $8.2 \pm 0.6$ \\
L-Octanoylcarnitine $(0.5 \mathrm{mM})$ & $9.4 \pm 1.2^{2}$ & $7.5 \pm 1.0$
\end{tabular}

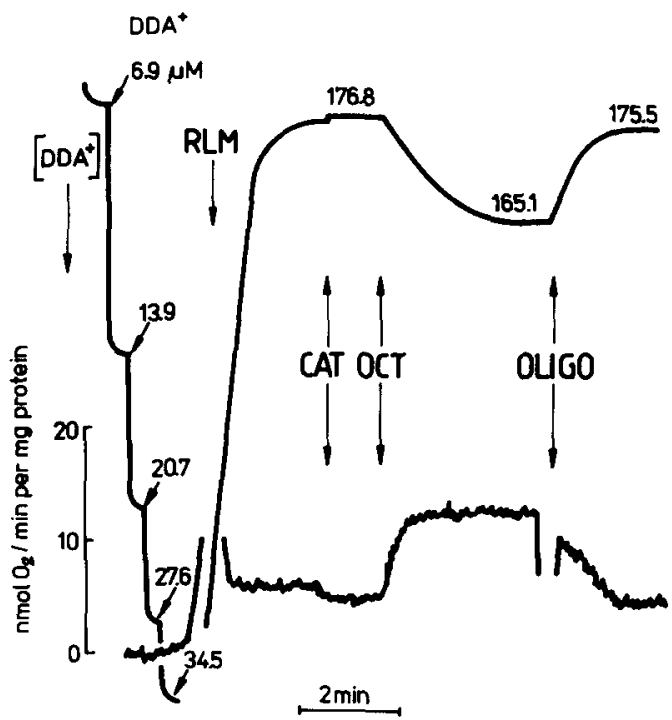

Fig. 1. Effect of octanoate on the membrane potential and respiration of rat liver mitochondria. The standard incubation medium comprised $5 \mathrm{mM}$ glutamate and $5 \mathrm{mM}$ malate. Further additions: dimethyldibenzylammonium chloride (DDA); mitochondria (RLM), $1.4 \mathrm{mg}$ protein $/ \mathrm{ml}$; carboxyatractyloside (CAT), $1 \mu \mathrm{M}$; octanoate (OCT), $0.5 \mathrm{mM}$; oligomycin (OLIGO) $5 \mu \mathrm{g} / \mathrm{ml}$. The upper trace shows the reading of the DDA-sensitive electrode (proportional to the logarithm of $\mathrm{DDA}^{+}$concentration). The numbers at the trace indicate the membrane potential in $\mathrm{mV}$. The lower trace shows the rate of oxygen uptake. 


\section{TABLE II}

EFFECT OF SHORT- AND MEDIUM-CHAIN LENGTH FATTY ACIDS ON PYRUVATE CARBOXYLATION

Rat liver mitochondria (3-4 mg protein $/ \mathrm{ml}$ ) were incubated as described under Materials and Methods. Sodium salts of the fatty acids were added to the final concentration of $0.5 \mathrm{mM}$. Mean values \pm S.D. for 3-6 different mitochondrial preparations are shown.

\begin{tabular}{lcc}
\hline Fatty acid added & \multicolumn{2}{l}{$\begin{array}{l}\text { Rate of pyruvate carboxylation } \\
\text { (nmol/min per mg protein) }\end{array}$} \\
\cline { 2 - 3 } & $\begin{array}{l}\text { no other } \\
\text { addition }\end{array}$ & $\begin{array}{l}0.5 \mathrm{mM} \text { L-octanoyl- } \\
\text { carnitine }\end{array}$ \\
\hline None & $14.7 \pm 1.3$ & $19.0 \pm 1.9$ \\
Acetate & $12.0 \pm 2.0$ & $9.5 \pm 0.9$ \\
Butyrate & $6.6 \pm 2.3$ & $6.3 \pm 1.6$ \\
Octanoate & $3.1 \pm 1.1$ & $3.9 \pm 0.4$ \\
\hline
\end{tabular}

tic decrease in the content of ATP and an increase of AMP. 2-Oxoglutarate prevented these changes (Table III) and abolished the inhibition of pyruvate carboxylation (not shown). Octanoylcarnitine neither decreased the rate of pyruvate carboxylation (on the contrary, it somewhat activated it, Table II) nor affected the level of intramitochondrial adenine nucleotides (not shown). Different degrees of inhibition of pyruvate carboxylation by acetate, butyrate and octanoate (Table II) were directly correlated with the effect of these fatty acids on the intramitochondrial content of ATP, as illustrated in Fig. 2.

\section{TABLE III}

\section{EFFECT OF OCTANOATE AND 2-OXOGLUTARATE ON} MITOCHONDRIAL ADENINE NUCLEOTIDE PATTERN

Mitochondria (3-4 $\mathrm{mg}$ protein $/ \mathrm{ml}$ ) were incubated in the presence of $10 \mathrm{mM}$ pyruvate with additions as indicated. Assuming that the leakage of adenine nucleotides from mitochondria was negligible, they were determined in the total incubation mixture without separating the mitochondria. The values are $\mathrm{nmol} / \mathrm{mg}$ protein and represent means \pm S.D. for four mitochondrial preparations.

\begin{tabular}{llll}
\hline Additions & ATP & ADP & AMP \\
\hline None & $6.2 \pm 0.5$ & $2.5 \pm 0.3$ & $3.8 \pm 0.6$ \\
Octanoate $(0.5 \mathrm{mM})$ & $2.0 \pm 0.4$ & $2.8 \pm 0.6$ & $8.6 \pm 1.0$ \\
2-Oxoglutarate $(5.0 \mathrm{mM})$ & $6.4 \pm 0.4$ & $2.2 \pm 0.1$ & $4.2 \pm 0.6$ \\
Octanoate + oxoglutarate & $5.1 \pm 0.7$ & $2.2 \pm 0.1$ & $4.8 \pm 0.1$ \\
\hline
\end{tabular}

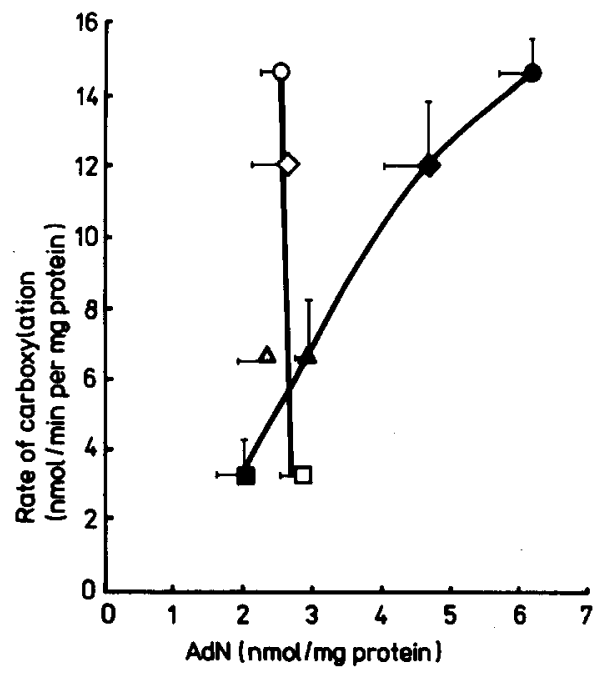

Fig. 2. Correlation between the rate of pyruvate carboxylation and the content of intramitochondrial ATP and ADP. Mitochondria (3-4 $\mathrm{mg}$ protein $/ \mathrm{ml}$ ) were incubated in the standard medium supplemented with $10 \mathrm{mM}$ pyruvate and $\mathrm{KH}^{14} \mathrm{CO}_{3}$, as described under Material and Methods. Fatty acids (sodium salts) were added to the final concentration of $0.5 \mathrm{mM}$. The symbols and the bars indicate the means \pm S.D. for 3-6 mitochondrial preparations. Open symbols, correlation with ADP; closed symbols, correlation with ATP; 0 , no fatty acid added; $\diamond \diamond$, acetate; $\Delta \Delta$, butyrate; $\square \mathbf{\square}$, octanoate.

Octanoate added to respiring mitochondria, in which pyrophosphatase (EC 3.6.1.1) was inhibited by fluoride, produced the accumulation of pyrophosphate (Fig. 3). This accumulation was not prevented by rotenone (if succinate was the respiratory substrate, Fig. 3B), indicating that it was not related to intramitochondrial oxidation of octanoate. On the other hand, however, pyrophosphate accumulation abolished, after a lag of a few minutes, the increased respiration produced by octanoate. This is shown in Fig. 4, where a steady decrease of the respiration, to the level observed before addition of octanoate, can be seen after addition of fluoride. This effect was not due to damage of the respiratory chain or of the coupling mechanism by the fluoride anion, since fluoride did not prevent a large burst of the respiration after addition of ADP. The rate of pyrophosphate accumulation, as shown in Fig. 3, amounted to about $2 \mathrm{nmol} / \mathrm{min}$ per $\mathrm{mg}$ protein, which does not fully account for the extra oxygen uptake produced by octanoate $(5.5 \mathrm{nmol}) \mathrm{O}_{2} / \mathrm{min}$ per $\mathrm{mg}$ protein, Table I), assuming the stoichiometry of 

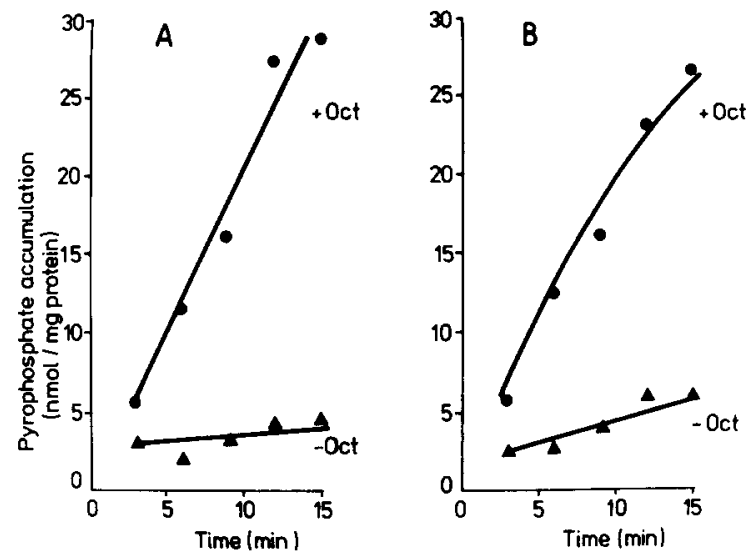

Fig. 3. Effect of octanoate on the production of pyrophosphate in liver mitochondria. Mitochondria (4-5 $\mathrm{mg}$ protein $/ \mathrm{ml}$ ) were incubated in the standard medium containing $5 \mathrm{mM}$ glutamate, $5 \mathrm{mM}$ malate and $20 \mathrm{mM}$ KF without (triangles) or with (circles) $0.5 \mathrm{mM}$ octanoate. In $\mathrm{B}$, the medium was additionally supplemented with $10 \mathrm{mM}$ succinate and $5 \mu \mathrm{M}$ rotenone. The symbols represent means for two different mitochondrial preparations.

$1 \mathrm{PP}_{\mathrm{i}}=1 \mathrm{AMP}=2 \mathrm{ADP}$ and a $P / O$ ratio of 3 . However, the values of Fig. 3 are underestimated, because pyrophosphate was determined in mitochondria separated from the medium by silicone oil filtration (because of interference by inorganic phosphate) and not in the whole incubation mixture. It has been recently suggested [26] that intramitochondrial pyrophosphate can be ex-

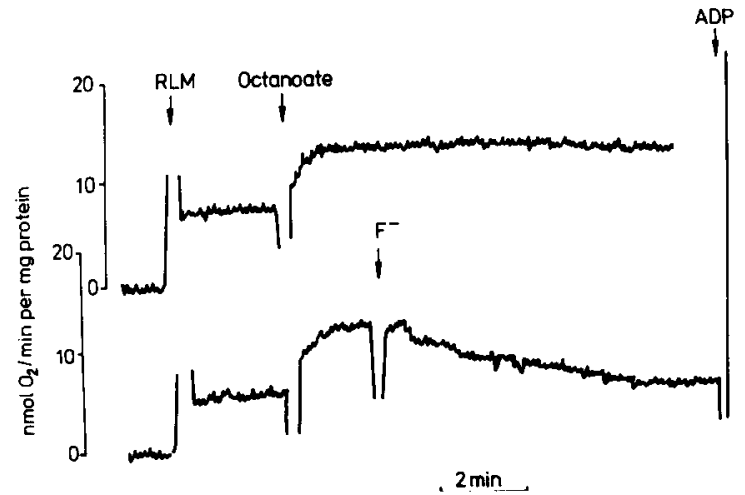

Fig. 4. Reversal of octanoate-induced stimulation of mitochondrial respiration by fluoride. Mitochondria (RLM), $1.7 \mathrm{mg}$ protein $/ \mathrm{ml}$, were added to the medium containing 5 $\mathrm{mM}$ glutamate and $5 \mathrm{mM}$ malate. Octanoate $(0.5 \mathrm{mM}), \mathrm{KF}(20$ $\mathrm{mM}$ ) and ADP (0.5 $\mathrm{mM})$ were added where indicated. The trace depicts the rate of oxygen uptake. changed not only with external adenine nucleotides (which were absent in our incubation medium), but also with inorganic phosphate. The discrepancy between an almost linear accumulation of pyrophosphate for $15 \mathrm{~min}$, shown in Fig. 3, and the return of oxygen-uptake rate to the 'normal' value after $5 \mathrm{~min}$ following fluoride addition (Fig. 4) can also be accounted for by different experimental conditions.

Octanoate is rapidly oxidized by isolated hepatocytes from starved animals. The rate of $\mathrm{O}_{2}$ uptake by hepatocytes was almost doubled by the addition of $3 \mathrm{mM}$ octanoate, whereas it was increased only by about $50 \%$ by $1 \mathrm{mM}$ oleate (Table IV). Both octanoate and oleate strongly stimulated glucose production from lactate plus pyruvate in isolated hepatocytes. However, the $\Delta$ glucose $/ \Delta$ $\mathrm{O}_{2}$ ratio was decreased by octanoate, whereas it was unchanged by oleate (Table IV).

In the presence of octanoate, but not of oleate, the total cellular ATP was decreased and AMP increased (Table IV). From data of Table IV the $[\mathrm{ATP}] \times[\mathrm{AMP}] /[\mathrm{ADP}]^{2}$ ratio can be calculated. In the absence of the fatty acid and in the presence of oleate it amounts to about 2.5 , which is somewhat higher that the equilibrium attained in the presence of adenylate kinase (EC 2.7.4.3). Upon addition of octanoate, this ratio increases to about 9 , indicating that the disequilibrium shifted towards ATP + AMP occurs in a compartment not accessible to adenylate kinase, presumably the mitochondrial matrix.

\section{Discussion}

Although the effects of octanoate on isolated mitochondria, such as increase of the resting-state respiration, decrease of the membrane potential and inhibition of pyruvate carboxylation, are typical for uncoupling, the sensitivity of the former two effects to oligomycin argues against true uncoupling, i.e., direct dissipation of the electrochemical proton gradient. All three effects can be therefore interpreted as resulting from the decrease of the intramitochondrial ATP level (pyruvate carboxylation, see also Ref. 19) or of the phosphorylating potential.

Although the stimulation of hepatic respiration has been observed with both octanoate and oleate 


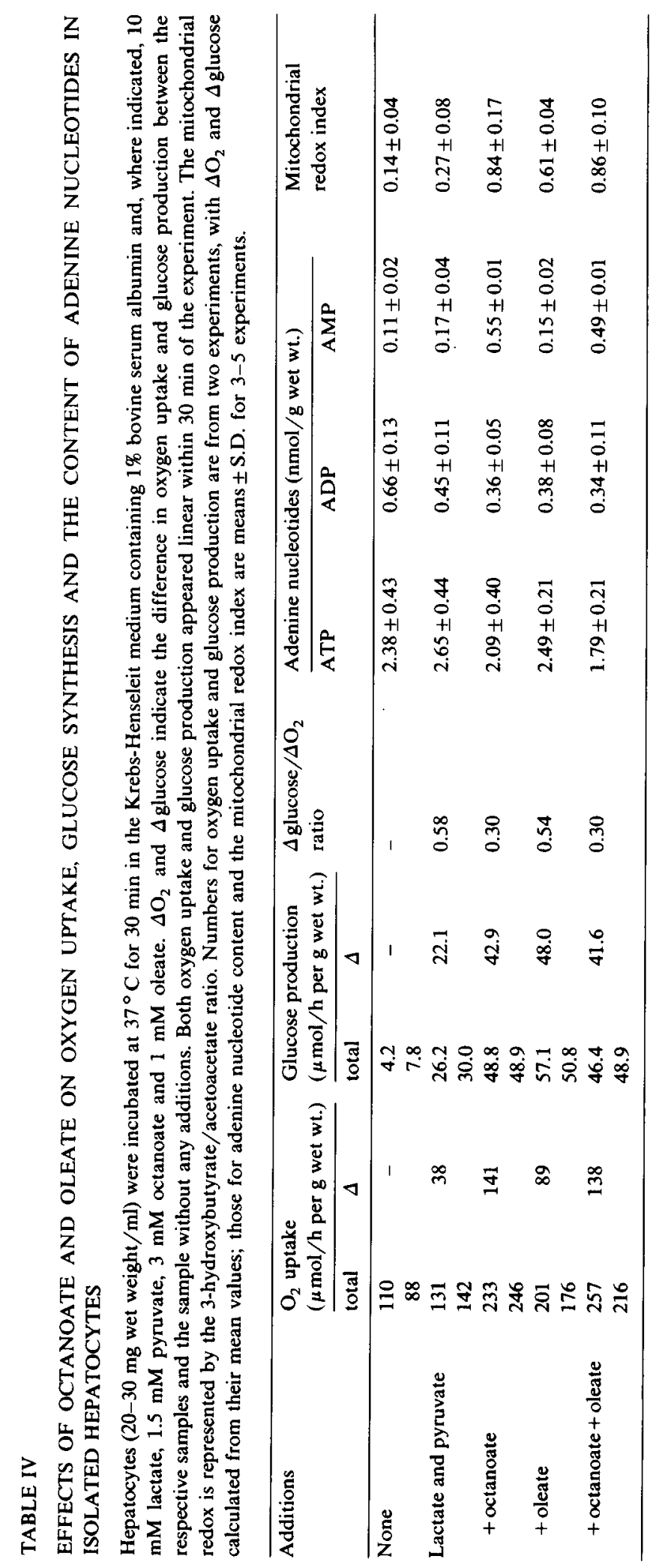


[1-8], the mechanism of this effect may not be the same for medium- and long-chain fatty acids, because they differ in two important aspects. Firstly, medium-chain fatty acids are esterified to corresponding acyl-CoA in the mitochondrial matrix, whereas long-chain acids are activated predominantly in the outer mitochondrial membrane and in the endoplasmic reticulum [27,28]. Secondly, medium-chain fatty acids (up to the chain length of 8 carbon atoms), in contrast to long-chain fatty acids (e.g., oleate), exert a small, uncoupling effect, if any [9,29].

The observation (Table I and Fig. 1) that the stimulation of oxygen uptake by isolated mitochondria is sensitive to oligomycin but not to carboxyatractyloside clearly points to the intramitochondrial localization of the ATP-utilizing process responsible for this stimulation. This is incompatible with a complete inhibition by carboxyatractyloside of the extra oxygen uptake in isolated hepatocytes, as described by Brand and Nobes [11]. However, Plomp et al. [10] observed only a partial sensitivity to carboxyatractyloside of the fatty acid-stimulated oxygen uptake in hepatocytes, which can be interpreted as indicating that the ATP-utilizing processes stimulated by fatty acids are located both in and outside mitochondria.

Because the oxidation of a fatty acid molecule feeds electrons partly to NAD and partly directly to the flavin component of the respiratory chain, the resulting $P / O$ ratio is about $2.5[30,31]$. In addition, activation of fatty acids (synthesis of acyl-CoA) consumes ATP and yields AMP, which is equivalent to the utilization of two high-energy phosphate bonds per molecule of fatty acid. Therefore, oxidation of fatty acids is less efficient than oxidation of NAD-linked substrates (for example, glutamate plus malate in Table I) in compensating the proton leak, which is regarded as the main energy-dissipating process responsible for the resting-state respiration [32]. This could, however, produce only a small increase of respiration when a fatty acid replaces an NAD-linked substrate, and not doubling the respiratory rate, as observed in isolated mitochondria (Table I) and hepatocytes (Table IV). Moreover, the potentiation of mitochondrial respiration was also observed when the substrate was succinate and the oxidation of fatty acid was prevented (Ref. 3 and this paper).

Stimulation of gluconeogenesis in isolated hepatocytes by medium- and short-chain fatty acids (Table IV) is difficult to reconcile with the inhibition of pyruvate carboxylation in mitochondria (Table II). It should, however, be remembered that the inhibition of pyruvate carboxylation can be observed only under State 4 conditions. In contrast to this, pyruvate carboxylation in State 3 is stimulated by low concentrations of fatty acids, including octanoate [19], although the absolute values of the carboxylation under these conditions are much lower. It is thus clear that fatty acids can influence pyruvate carboxylation by various mechanisms. One of these could be the allosteric activation by acetyl-CoA, which was probably responsible for the activatory effect of octanoylcarnitine as shown in Table II. For the purpose of the present paper it is, however, important to point out that under restingstate conditions (in the absence of external ATP) the rate of pyruvate carboxylation was correlated with the intramitochondrial level of ATP (Fig. 2).

In isolated hepatocytes, in addition to increased oxygen uptake also described by other authors [6-8], we observed a decreased 'efficiency' of gluconeogenesis as expressed by a decrease of the $\Delta$ glucose/ $\Delta$ oxygen ratio (Table IV). Although this ratio represents a correlation and not necessarily a causal relationship, its decrease may be interpreted as indicating a partial dissipation of energy of oxidative phosphorylation. However, the mitochondrial redox index was not lowered (in fact, it was considerably increased) by octanoate or oleate, arguing against the true uncoupling effect of these fatty acids at the concentrations used.

All these facts, together with intramitochondrial accumulation of AMP (which can, however, be prevented by 2-oxoglutarate) and pyrophosphate (see also Ref. 33), allow us to propose that the utilization of intramitochondrial ATP, responsible for the 'uncoupling-like' effect of medium-chain fatty acids, is due to intramitochondrial hydrolysis of acyl-AMP and acyl-CoA. Such an explanation was already suggested by Debeer et al. [3], though it has not been taken into due consideration subsequently.

A rapid hydrolysis of hexanoyl-AMP by an 
enzyme extracted from hog liver particulate fraction (mitochondria) was already reported three decades previously by Jencks and Lipmann [34] and a highly active medium-chain acyl-CoA hydrolase (EC 3.1.2.?) was described by Osmundsen and Sherratt [35] for rat liver mitochondria. A rapid cycling of acetate and acetyl-CoA in rat hepatocytes has been described more recently [36]. It can be therefore inferred that in the presence of medium- or short-chain fatty acids, a futile cycle is initiated in the mitochondrial matrix, consisting of the formation of acyl-CoA by medium-chainspecific acyl-CoA synthetase (EC 6.2.1.2), with acyl-AMP as intermediate [37], and subsequent hydrolysis of acyl-AMP and acyl-CoA. As a result, ATP is utilized and AMP accumulates. This produces a decrease in the membrane potential (because of a decrease in the intramitochondrial phosphorylation potential) and, as a consequence, an increase in the resting-state respiration. Inhibition of pyruvate carboxylation in mitochondria and the decrease of the $\Delta$ glucose $/ \Delta \mathrm{O}_{2}$ ratio in intact hepatocytes are further consequences. ATP can be regenerated by the following reactions:

$\mathrm{GTP}+\mathrm{AMP} \rightleftharpoons \mathrm{GDP}+\mathrm{ADP}$

$\mathrm{GDP}+\mathrm{ATP} \rightleftharpoons \mathrm{GTP}+\mathrm{ADP}$

followed by phosphorylation of ADP to ATP in oxidative phosphorylation. HOwever, since reactions (1) and (2) in the mitochondrial matrix are rather slow [38], an increased steady-state level of AMP is observed (Tables III and IV). This explanation is also compatible with intramitochondrial accumulation of pyrophosphate when pyrophosphatase is inhibited (Fig. 3), and with inhibition of the extra oxygen uptake by accumulating pyrophosphate (Fig. 4). In the latter case, formation of acyl-CoA is prevented.

One has to question immediately the possible physiological importance of such a futile cycle dissipating energy. A plausible explanation is that operation of this cycle has been deduced from experiments with abnormally high concentrations of medium chain fatty acids, either in perfusates or incubation media, which rarely occur in the portal vein blood under normal feeding conditions. The $K_{\mathrm{m}}$ of medium-chain acyl-CoA hydrolase for hexanoyl-CoA is $0.5 \mathrm{mM}$ [35]. Free medium-chain acyl-AMP appears in substantial amounts as an intermediate of fatty acid activation only in the presence of large excess of its substrates [39]. It can be therefore concluded that the futile hydrolysis of acyl-CoA and, possibly, acyl-AMP becomes significant in liver mitochondria in situ only under conditions of a large supply of medium- or short-chain fatty acids. Under such conditions this mechanism may play an important role as a 'safety valve', preventing intramitochondrial free $\mathrm{CoA}$ from decreasing drastically, thus enabling undisturbed operation of the tricarboxylic acid cycle.

\section{Acknowledgements}

We thank Mrs. H. Goldammer and Mrs. U. Meyer for skilful technical assistance. This work was supported in part by Grant CPBP 04.01 of the Polish Academy of Sciences.

\section{References}

1 Williamson, J.R., Scholz, R. and Browning, E.T. (1969) J. Biol. Chem. 244, 4617-4627.

2 Williamson, J.R., Scholz, R., Browning, E.T., Thurman, R.G. and Fukami, M.H. (1969) J. Biol. Chem. 244, 5044-5054.

3 Debeer, L.J., Mannaerts, G. and De Schepper, P.J. (1974) Eur. J. Biochem. 47, 591-600.

4 Scholz, R., Schwabe, U. and Soboll, S. (1984) Eur. J. Biochem. 141, 223-230.

5 Soboll, S., Gründel, S. and Scholz, R. (1984) Eur. J. Biochem. 141, 231-236.

6 Van der Meer, R., Akerboom, T.P.M., Groen, A.K. and Tager, J.M. (1978) Eur. J. Biochem. 84, 421-428.

7 Berry, M.N., Clark, D.G., Grivell, A.R. and Wallace, P.G. (1983) Eur. J. Biochem. 131, 205-214.

8 Berry, M.N., Gregory, R.B., Grivell, A.R. and Wallace, P.G. (1983) Eur. J. Biochem. 131, 215-222.

9 Wojtczak, L. (1976) J. Bioenerg. Biomembr. 8, 293-311.

10 Plomp, P.J.A.M., Van Roermund, C.W.T., Groen, A.K., Meijer, A.J. and Tager, J.M. (1985) FEBS Lett. 193, 243-246.

11 Brand, M.D. and Nobes, C.D. (1986) Biochem. Soc. Trans $14,1200-1201$.

12 Schönfeld, P. and Petzold, D. (1986) 4th European Bioenergetics Conference, Prague, Reports, p. 372.

13 Steinbrecht, I. and Kunz, W. (1970) Acta Biol. Med. German. 25, 731-747.

14 Berry, M.N. and Friend, D.S. (1969) J. Cell. Biol. 43, 506-520.

15 Geelen, M.J.H. and Gibson, D.M. (1976) in Use of Isolated Cells and Kidney Tubules in Metabolic Studies (Tager, 
J.M., Söling, H.D. and Williamson, J.R., eds.), pp. 219-232, North-Holland, Amsterdam.

16 Krebs, H.A. and Henseleit, K. (1932) Hoppe-Seyler's Z. Physiol. Chem. 210, 33-66.

17 Krebs, H.A., Cornell, N.M., Lund, P. and Hems, R. (1974) in Regulation of Hepatic Metabolism (Lundquist, F. and Tygstrup, N., eds.), pp. 726-750, Munksgaard, Copenhagen.

18 Schild, L. and Schönfeld, P. (1986) Biomed. Biochem. Acta $45,1215-1225$

19 Wojtczak, A.B., Lenartowicz, E., Rodionova, M.A. and Duszyński, J. (1972) FEBS Lett. 28, 253-258.

20 Lamprecht, W. and Trautschold, J. (1970) in Methoden der Enzymatischen Analyse (Bergmeyer, H.U., ed.), pp. 2024-2033, Akademie-Verlag, Berlin.

21 Jaworek, D., Gruber, W. and Bergmeyer, H.U. (1970) in Methoden der Enzymatischen Analyse (Bergmeyer, H.U., ed.), pp. 2051-2055, Akademie-Verlag, Berlin.

22 Mellanby, J. and Williamson, D.H. (1970) in Methoden der Enzymatischen Analyse (Bergmeyer, H.U., ed.), pp. 1776-1779, Akademie-Verlag, Berlin.

23 Williamson, D.H. and Mellanby, J. (1970) in Methoden der Enzymatischen Analyse (Bergmeyer, H.U., ed.), pp. 1773-1775, Akademie-Verlag, Berlin.

24 Bergmeyer, H.U. and Bernt, E. (1970) in Methoden der Enzymatischen Analyse (Bergmeyer, H.U., ed.), pp. 1172-1181, Akademie-Verlag, Berlin.

25 Grindey, G.B. and Nichol, A.C. (1970) Anal. Biochem. 33, 114-119.
26 Halestrap, A.P. and Davidson, A.M. (1989) in Mitochondrial Transport Proteins (Azzi, A., Nalęcz, K.A., Nalęcz, M.J. and Wojtczak, L., eds.), Springer-Verlag, Heidelberg, in press.

27 Aas, M. and Bremer, J. (1968) Biochim. Biophys. Acta 164, 157-166.

28 Aas, M. (1971) Biochim. Biophys. Acta 231, 32-47.

29 Pressman, B.C. and Lardy, H.A. (1956) Biochim. Biophys. Acta 21, 458-466.

20 Hird, F.J.R. and Weidemann, M.J. (1966) Biochem. J. 98, 378-388.

31 Bremer, J. and Davis, E.J. (1972) Biochim. Biophys. Acta 275, 298-301

32 Nicholls, D.G. (1974) Eur. J. Biochem. 50, 305-315.

33 Batenburg, J.J. and Van den Bergh, S.G. (1972) Biochim. Biophys. Acta 280, 495-505.

34 Jencks, W.P. and Lipmann, F. (1957) J. Biol. Chem. 225, 207-223.

35 Osmundsen, H. and Sherratt, H.S.A. (1975) FEBS Lett. 55, $38-41$.

36 Jessop, N.S., Smith, G.H. and Crabtree, B. (1986) Biochem. Soc. Trans. 14, 146-147.

37 Webster, Jr., L.T. and Campagnari, F. (1962) J. Biol. Chem. 237, 1050-1055.

38 Krebs, H.A. and Wiggins, D. (1978) Biochem. J. 174, 297-301

39 Webster, L.T., Jr. (1963) J. Biol. Chem. 238, 4010-4015. 